\title{
Out-ward monitoring of neurorehabilitation in subacute stroke
}

\author{
Silvia I. Franco-Pastrana \\ Felipe Orihuela-Espina \\ Patrick Heyer-Wollenberg \\ Javier Herrera-Vega \\ Luis Enrique Sucar \\ (silviafp,f.orihuela-espina, patrickhey, \\ vega,esucar)@inaoep.mx \\ Instituto Nacional de Astrofísica, \\ Óptica y Electrónica (INAOE) \\ Tonantzintla, Puebla, Mexico
}

\author{
Luis Castrejón \\ fisiatriapuebla@gmail.com \\ Hospital Universitario Benemérita \\ Univerisdad Autónoma de Puebla \\ Puebla, Puebla, Mexico
}

\author{
Mayela Saucedo \\ Lorena Palafox \\ Jorge Hernández-Franco \\ mayesaucedo@hotmail.com \\ licto.palafoxlorena@gmail.com \\ jhfranco@medicapolanco.com \\ Instituto Nacional de Neurología y \\ Neurocirugía \\ Mexico City, Mexico
}

\begin{abstract}
Validated clinical scales of functional dexterity of stroke patients provide only sparse and controlled observations of the neurorehabilitation process. Continuous naturalistic monitoring the progress during the rehabilitation therapy of out-patients remains unsatisfactorily resolved. We propose a new indicator of functional performance capitalizing on smart devices and the cloud services. The new index of limb usage balance (ILUB) derived from accelerometry data, provides an objective scalar that it is easy to estimate and interpret, and adequate for continuous uncontrolled monitoring. Three studies for the verification and validation of the index and accompanying platform are presented. We show that the index is unbiased to handedness dominance and provide preliminary evidence that it is appropriate for patients even during the subacute stage. The new index can complement clinical scales to observe the patient progress.
\end{abstract}

CCS Concepts $\quad$-Social and professional topics $\rightarrow$ Medical technologies; $\bullet$ Human-centered computing $\rightarrow U b i q-$ uitous and mobile computing; Ubiquitous and mobile computing systems and tools; • Applied computing $\rightarrow$ Health care information systems.

Keywords stroke, neurorehabilitation, home monitoring, index of limb usage, accelerometry, cloud computing

\footnotetext{
Permission to make digital or hard copies of all or part of this work for personal or classroom use is granted without fee provided that copies are not made or distributed for profit or commercial advantage and that copies bear this notice and the full citation on the first page. Copyrights for components of this work owned by others than the author(s) must be honored. Abstracting with credit is permitted. To copy otherwise, or republish, to post on servers or to redistribute to lists, requires prior specific permission and/or a fee. Request permissions from permissions@acm.org. Pervasive Health '19, May 20-23, 2019, Trento, Italy

(c) 2018 Copyright held by the owner/author(s). Publication rights licensed to ACM.

ACM ISBN xxx-x-xxxx-xxxx-x/xx/xx...\$xx.00

https://doi.org/xx.xxxx/xxxxxxx.xxxxxxx
}

\section{ACM Reference Format:}

Silvia I. Franco-Pastrana, Felipe Orihuela-Espina, Patrick HeyerWollenberg, Javier Herrera-Vega, Luis Enrique Sucar, Luis Castrejón, Mayela Saucedo, Lorena Palafox, and Jorge Hernández-Franco. 2018. Out-ward monitoring of neurorehabilitation in subacute stroke. In Pervasive Health 2019: 13th EAI International Conference on Pervasive Computing Technologies for Healthcare, May 20-23, 2019, Trento, Italy. ACM, New York, NY, USA, 4 pages. https://doi.org/xx.xxxx/ xxxxxxx.xxxxxxx

\section{Introduction}

Monitoring the patient while out-ward has been hindered by a number of issues (cost, lack of pervasive sensing platform, etc) until recently. Existing clinical scales [3] for the assessment of motor function only provide highly controlled instantaneous snapshots along the long rehabilitatory process failing to give the clinician information about the impact of the therapy once the patient leaves the ward. There is a need for a complementary continuous ecological indicator of the therapy benefit to the patient during this critical period of rehabilitation. Desirable properties of such an indicator should include (i) applicable in highly unrestricted settings, (ii) ubiquitously accessible, e.g. ambulatory, (iii) can be automated so that estimation is made without human intervention, (iv) easy to interpret not only for a clinician but also for the patient and its social circle, and (v) sensitive to neurorehabilitation goals (i.e. returning the patient its functional independence in everyday life). A satisfactory solution has not yet been developed to this problem.

This research main contribution is the development of an indicator that fulfills the above wish-list, and its supporting platform for operationalization. The new index measures how much the paretic side is used with regard to a population baseline (the main contribution) and we believe can complement current clinical evaluations. The application implements the index and is realized by using two smartwatches and one smartphone. The former sense arm activity, and the latter concentrates data and upload it to the cloud. 
Real time measurements can be read directly on the phone, or through the Web. Three studies have been or are being carried out on both, healthy and stroke volunteers, for the validation of the index and its supporting platform.

\section{Related work}

In an attempt to alleviate the burden of patient assessment on the clinical team, and facilitate such assessment, there have been efforts to automatize the scoring of validated clinical scales of motor dexterity [2]. These efforts maintain in as much as possible the controlled conditions under which the original scale was meant to be employed. The rise of robotic and virtual rehabilitation alternatives [7] permitted more flexible therapy administration and automatic objective evaluation of many indicators related to functional progress, but cost and questioned translational value [4] are hindering widespread adoption. Wearable solutions $[1,6]$ have enable the automatic and ubiquitous continuous monitoring of activity of neurorehabilitation patients with low-cost, but the focus of state-of-the-art solutions appears to be the discrimination of tasks, rather than functional assessment.

\section{Methods}

\subsection{Index of limb usage balance (ILUB)}

We depart from the existing laterality index (LI) [5] which provides a metric of the balance between two service providers, expressed by $L I=(R-L) /(R+L)$, with $R$ and $L$ expressing the intensity of the response by each provider. Reexpressing the LI in terms of accelerometry data results in Equation 1:

$$
L I a c c=\frac{R-L}{R+L}=\frac{R A c c_{x y z}-L A c c_{x y z}}{R A c c_{x y z}+L A c c_{x y z}}
$$

where now $R$ and $L$, here indicated as $R A c_{x y z}$ and $L A c c_{x y z}$ respectively, are operationalized in terms of the area under the curve (AUC) of the magnitude of $<x[t], y[t], z[t]>\mathrm{vec}-$ tors with the $x[t], y[t]$ and $z[t]$ values corresponding to data from two accelerometers, and $[t]$ expressing discrete sampling in time. Equation 1 intentionally hides the temporal dependence of LIacc. The practical implementation, requires some windowing of the incoming stream of accelerometer data permiting calculation of LIacc at some discrete times. The implications of this parameterization is beyond this paper and here we shall use an arbitrary fix window.

Most humans exhibit dominance from one side of its brain (translated to a contralateral behavioural dominance), hence, the LI would be difficult to interpret. Hence, we define the index of limb usage balance (ILUB) according to Equation 2:

$$
I L U B_{\text {subj }(H)}=\frac{\operatorname{LIacc}_{\text {subj }(H)}}{\operatorname{LIacc}_{\text {population }(H)}}
$$

with $H$ some measure of handedness for a certain subject subj. In our case, the $H$ is made equal to the outcome of the
Edinburgh Handedness Inventory (EHI) [5], but other indicators can be used. The EHI, originally developed to measure hand laterality, derives a laterality quotient alike Equation 1 from the scores obtained by the subject at a series of twenty tasks . Of course, the discrete temporal dependence in Equation 1 propagates here, and $I L U B_{s u b j[H]}$ fluctuates in time. If preferred, we can make this explicit, i.e. $I L U B_{s u b j(H)}[t]$

The ratio in Equation 2 removes the dominance bias facilitating interpretation. A ILUB:

- $>1$ : The participant uses its right limb more than its left limb (with respect to the healthy population)

- $\approx 1$ : The participant has not substantially modified its behaviour (with respect to the healthy population)

- $<1$ : The participant uses its left limb more than its right limb (with respect to the healthy population)

This can later straight-forwardly be re-expressed in terms of its affected limb and subject's dominance prior to stroke.

\subsection{SmartHealth App on FI-WARE}

FI-WARE (https://www.fiware.org/) is an open source framework for cloud computing. It defines standard data models and enablers to connect smart devices through the internet of things (IoT). The Smart Health application developed uses existing FI-WARE data model and enablers to gather the data. The application itself implements the index and is realized by using two smartwatches and one smartphone. We employed Huawei Watch 2 LEO BX9 smartwatches and a Motorola Moto G5 Plus smartphone. An android application was developed in a client/server architecture to collect the data. A running service in each smartwatch collects data from all the built in sensors detected by the Android OS. This includes; accelerometers, gyroscope, lighting, magnetic field, and heart rate. The data collected by the sensors is transmitted over Bluetooth asynchronously to the smartphone. The information collected on the smartphone is then combined by the app, into a FI-WARE data model and uploaded to a cloud storage. Once the data is uploaded, the FI-WARE back-end uses a combination of the Context-Broker for receiving and processing the data, and QuantumLeap that permanently saves the data for visualization and future querying. Real time measurements of every sensor can be visualized directly on the phone main application, and/or off-line in the user's preferred graphical rendering software (e.g. ggplot) or Grafana (https://grafana.com/).

Figure 1 schematically depict the hardware and FI-WARE software architecture of the smart health application for neurorehabilitation. For the purpose of estimating the ILUB the exact location of the accelerometers is irrelevant as long as there is some degree of symmetry but distal locations such as the wrist are likely good proxies of limb usage. Moreover, minor deviations are negligible in the long term.

Although the watches may acquired data at $50 \mathrm{~Hz}$, neurorehabilitatory processes last for years and variations in 


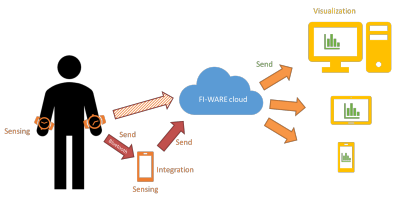

(a) Hardware architecture

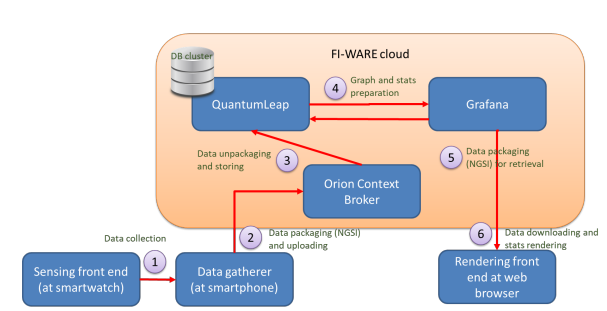

(c) Software architecture

Figure 1. Schematic depiction of the architecture of the smart health application for neurorehabilitation

motor dexterity are extremely slow. In this sense, a high sampling rate is considered unnecessary and consequently sampling rate was downrated to $0.5 \mathrm{~Hz}$. A moving average low pass filter was applied with window sized 30 samples and uniform weight among samples. Accelerometry data was not corrected for gravity. This introduces a shift in absolute measurements but because our endpoints are relative magnitudes (Equation 2) this shift will cancel out.

\section{Experiments}

Three studies have been (or are being) conducted with different purposes; (i) a proof of concept, (ii) ILUB verification and (iii) a clinical pilot. The proof of concept was approved by project internal ethical committee. The clinical study underwent approval by the involved hospitals ethical committees. The verification study was not subject to ethical approval. All three required informed consent from volunteers, and were conducted in full respect of the Helsinki declaration.

\subsection{Proof of concept}

The feasibility of the application was tested (for instance regarding the battery consumption or the detection of critical bugs) in order to establish limitations and/or improvements for a subsequent trial on real patients. The study was carried out at the Instituto Nacional de Astrofísica, Óptica y Electrónica (INAOE) main campus in Puebla, Mexico as well as the participants home. For this proof of concept, only healthy participants were recruited. Twenty volunteers were enrolled. The study was designed as a cross-sectional study (1 session per experimental unit), where the session involves a week of data collection through the application. We considered "normal" full day usage (this includes use from wake up until bedtime with the expected breaks for grooming, showering, toileting, charging of the devices, etc). A minimum of 4h per day was requested to be considered for analysis. At least data from 3 days was required, of which at least 2 must be usable for analysis.

\subsection{ILUB verification}

The second study was conceived to verify and establish the face validity of the $I L U B$ operationalized as per Equation 2 as a measure of functional limb usage. We asked participants to execute a series of task for which the laterality (or lack of) of the tasks has been pre-established from the population. Their $I L U B$ was recorded during task execution and compared against the expected trend. The activities were; throwing a ball into a cup, hitting a ball with a golf club, hitting a ball with a cricket bat, put coins in a piggy bank, solving a puzzle, separate cards in a deck, spelling with foam letters, knot tying, scissor cutting, grooming a doll, and "teaching" the doll to get ready and eat. The principal endpoint is the ILUB.

Ten healthy subjects, distinct from those who participated in the first study, were recruited from INAOE population for carrying out the activities proposed. Volunteers were not instructed to use a particular hand to execute the activities. This verification expects to obtain $I L U B$ values deviating from 0 for lateralised tasks, and values closer to 0 for balanced tasks. At the end of the execution of the activities, the EHI score was calculated for the volunteers.

\subsection{Clinical pilot}

A multicenter clinical pilot is undergoing at the Instituto Nacional de Neurología and Neurocirugía (INNN) in Mexico City and at the Hospital Universitario de la Benemérita Universidad Autónoma de Puebla (HU-BUAP). Patient are being recruited since June 2018. Thus far 3 subjects have been enrolled. The out-ward monitoring of stroke patients occurred while they receive a virtual rehabilitation therapy Gesture Therapy [7] combined with physical therapy at the hospital where they attend rehabilitation sessions 3 times a week for 45 minutes. Functional assessment using the FuglMeyer scale was collected during the therapeutic sessions but it is not reported here.

\section{Results and Discussion}

\subsection{Proof of concept}

The tests carried out during this study permitted us to debug and polish the original app for the subsequent studies. The poblational LI as a function of the EHI score is shown in Figure $2 \mathrm{a}$. The bias correction due to handedness of the ILUB is evident in Figure 2b. This confirms that the ILUB can be interpreted without consideration for the subject handedness. Under hemiparesis, the subject will be pushed away from the neutral ILUB score whether to the right $(\operatorname{ILUB}>>1)$ or to the left $(I L U B<<1)$, and as s/he recovers, s/he will be 


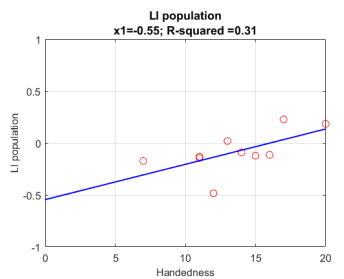

(a) Poblational LI

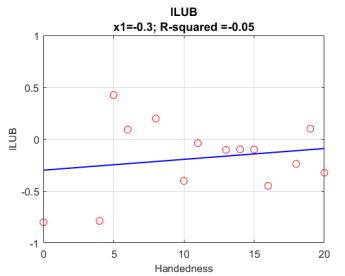

(b) ILUB
Figure 2. (a) Poblational laterality index as a function of the EHI score. Each point represents the average of observed values for a given handedness score. (b) Poblational ILUB as a function of the EHI score. $x 1$ is the slope of the regression.

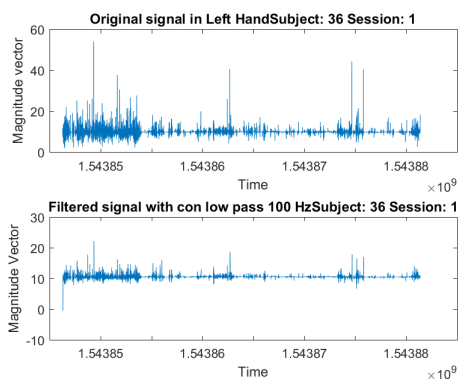

Figure 3. Top: Magnitude vectors estimated from the accelerometer data. Bottom: Its moving averaged filtered counterpart. Timestamps are sequential but otherwise labelled according to an arbitrary 0 .

returning gradually to the neutral value. This hypothesis, still to be validated, seems nonetheless plausible.

\subsection{ILUB verification}

Data from 12 activities from 10 volunteers was collected. 120 observations were collected that after parsing and cleaning left us with 188 data chunks. Fourier analysis of the recordings (not shown) were used to confirm tasks major rythms and these were matched to videos of the data sessions. Then, the respective estimated $I L U B$ were qualitatively matched against the foreknown hand dominance of the task. Confirmation of these facts of hand dominance give us confidence that the chosen testbed is appropriate for our verfication purposes. Further quantitative analysis is still ongoing.

\subsection{Clinical pilot}

Age of the patients is 65-85 years old, 1 man and 2 women, 2 with an hemorrhagic event and 1 with ischemic event. We already had an abandonment. Exemplary accelerometry data from patients and the effect of filtering is shown in Figure 3. Space constraints does not permits us to provide further details at this point.
Limitations. Recruiting rate is limited by our current infrastructure ( 5 smartwatches and 3 smartphones). Tremors are not considered. The use of platform by third people (e.g. at home) or some clinical parameters of interest (time from stroke, location of stroke, etc) are not controlled in these studies. We lack a baseline of activity limb usage for patients.

\section{Conclusions}

We have presented a new index for monitoring limb usage balance that we propose as a suitable indicator for continuous naturalistic monitoring of patient progress through the neurorehabilitation process. The index is calculated from accelerometry data, and for this, we have presented a cloudbased smart application to gather and handle such data. The index has been verified and validated and we have obtained preliminary clinical evidence of its translational value. These preliminary results hints that the index has good face validity, although our ongoing analysis will reveal further insights.

\section{Acknowledgments}

The FI-WARE data model was developed by J. Favela and N. Hernández-Cruz. Research funded by the EU (H2020-ICTINT-38-723174) and by Secretaría de Educación, Ciencia, Tecnología e Innovación de la Ciudad de México SECITI/042/2018 (INGER-DI-CRECITES-001-2018) "Red Colaborativa de Investigación Traslacional para el Envejecimiento Saludable de la Ciudad de México" (RECITES).

\section{References}

[1] Valerie AJ Block, Erica Pitsch, Peggy Tahir, Bruce AC Cree, Diane D Allen, and Jeffrey M Gelfand. 2016. Remote physical activity monitoring in neurological disease: a systematic review. PloS one 11, 4 (2016), e0154335.

[2] Patrick Heyer, Luis R. Castrejón, Felipe Orihuela-Espina, and Luis Enrique Sucar. 2017. Automation of motor dexterity assessment. In 15th IEEE Conference on Rehabilitation Robotics (ICORR 2017), Farshid Amirabdollahian, Etienne Burdet, and Lorenzo Masia (Eds.). IEEE, London, United Kingdom, 521-526.

[3] Peter Langhorne, Julie Bernhardt, and Gert Kwakkel. 2011. Stroke rehabililtation. Lancet 377 (MAY 2011), 1693-1702.

[4] Kate E. Laver, George Stacey, Susie Thomas, Judith E. Deutsch, and Maria Crotty. 2011. Virtual reality for stroke rehabilitation. Cochrane Database of Systemic Reviews 9 (2011), 70 pp. DOI : http://dx.doi.org/10. 1002/14651858.CD008349.pub2

[5] R. C. Oldfield. 1971. The assessment and analysis of handedness: the Edinburgh inventory. Neuropsychologia 9, 1 (1971), 97-113. DOI : http://dx.doi.org/10.1016/0028-3932(71)90067-4

[6] Franchino Porciuncula, Anna Virginia Roto, Deepak Kumar, Irene Davis, Serge Roy, Conor J Walsh, and Louis N Awad. 2018. Wearable Movement Sensors for Rehabilitation: A Focused Review of Technological and Clinical Advances. PM\&R 10, 9 (2018), S220-S232.

[7] Luis Enrique Sucar, Felipe Orihuela-Espina, Roger Luis Velázquez, David J. Reinkensmeyer, Ronald Leder, and Jorge Hernández-Franco. 2014. Gesture Therapy: An upper limb virtual reality-based motor rehabilitation platform. IEEE Transactions on Neural Systems and Rehabilitation Engineering 22, 3 (2014), 634-643. DOI : http://dx.doi.org/ 10.1109/TNSRE.2013.2293673 